\title{
Engineering couplings for exciton transport using synthetic DNA scaffolds
}

Stephanie M. Hart ${ }^{1 \dagger}$, Wei Jia Chen ${ }^{1 \dagger}$, James L. Banal ${ }^{2 \dagger}$, William P. Bricker ${ }^{2,3}$, Amro Dodin ${ }^{2}$, Larysa Markova $^{4}$, Yuliia Vyborna ${ }^{4}$, Adam P. Willard ${ }^{1}$, Robert Häner ${ }^{4}$, Mark Bathe ${ }^{2 *} \&$ Gabriela S. Schlau-Cohen $^{1 *}$

${ }^{1}$ Department of Chemistry, Massachusetts Institute of Technology, Cambridge, MA 02139, USA

${ }^{2}$ Department of Biological Engineering, Massachusetts Institute of Technology, Cambridge, MA 02139, USA

${ }^{3}$ Department of Chemical and Biological Engineering, University of New Mexico, Albuquerque, NM 87131, USA (Present address)

${ }^{4}$ Department of Chemistry and Biochemistry, University of Bern, Freiestrasse 3, CH-3012 Bern, Switzerland 
Control over excitons enables electronic energy to be harnessed and transported for applications in light harvesting and molecular electronics. Such control requires nanoscale structural precision over the molecular components. Natural light-harvesting systems achieve the requisite precision with sophisticated protein machinery, which has been challenging to replicate synthetically. Here, we introduce a DNA-based platform that arranges cyanine chromophores with nanoscale precision for the construction of tunable excitonic systems. We synthesize DNA strands with inserted chromophores, produce chromophore-DNA nanostructures, and characterize them with ultrafast and single-molecule spectroscopy and structure-based modeling. This approach establishes simultaneous and independent control over the coupling among the chromophores and between the chromophores and the environment, using the properties of the DNA scaffold. With this control, we demonstrate that the coupling between the chromophores and the environment can enhance the exciton transport efficiency. The enhanced efficiency shows the power of the environment in driving exciton dynamics, a property previously underutilized experimentally. Control over excitons as reported here offers a path towards the design and fabrication of nanophotonic devices.

The ability of natural systems to harness and control the flow of electronic energy is essential for nearly all life on Earth. ${ }^{1+4}$ Photosynthetic light-harvesting systems have evolved specifically to exert this control using networks of electronically-coupled chromophores positioned with nanoscale precision. ${ }^{[5-10}$ The organization of these chromophore networks effectively guides the dynamics of excitons, i.e., bound electron-hole pairs created upon optical excitation. Measurements of excitons within these networks have revealed complex dynamical properties that are not captured by simple models, 11 which has led to active debates about the fundamental mechanism of exciton transport. $\frac{12,20}{20}$ Understanding these mechanisms has been challenging because they depend on the interplay of multiple electrodynamic couplings, which are not easily disentangled. This limited understanding has, in turn, limited the design of molecular systems for energy harvesting, transport, and quantum information processing.

The photosynthetic light-harvesting machinery achieves nanoscale precision over chromophore positioning via the structure of protein assemblies. The chromophores are embedded within proteins at specific distances and relative orientations, which determine the electrodynamic couplings both among the chromophores (electronic coupling) and also between the chromophores and their surrounding environment 
(system-bath coupling). The properties of these couplings, including the strength and topology of the electronic coupling network and the relative magnitude of the system-bath coupling, determine the dynamics of the excitons. Subtle variations in chromophore positioning can dramatically alter how excitons evolve, for example by changing their tendency to delocalize or shifting the balance between coherent and incoherent modes of transport. Precise nanoscale control over the molecular architecture is required to enable systematic examination of electronic and system-bath couplings and, therefore, of exciton dynamics.

Nanoscale control over chromophore position is challenging in proteins due to their structural complexity and limitations in mutagenesis. $\left.{ }^{21}-23\right]$ Instead, synthetic model systems that position chromophores have been developed including metal-organic frameworks, ${ }^{24}$ viral proteins,,$\sqrt{25-\sqrt{27}}$ self-assembled molecular aggregates, 28 and conjugated polymers. ${ }^{32}$ However, these systems lack a synthetic handle for electronic coupling. Covalently-linked, small-molecule dimers are more tractable alternatives through which electronic coupling has been systematically varied $\sqrt[33]{34}$ In the regime of strong electronic coupling, however, the influence of nuclear motion has been more challenging to vary, and thus system-bath coupling has only been studied to a limited extent $\frac{\sqrt[35]{36}}{5}$ Furthermore, small-molecule dimers have a fixed spatial relationship due to the rigid organic linkers that connect them, limiting the ability to control the number of chromophores and to integrate these systems to form higher-order excitonic assemblies. DNA-templated chromophores have recently emerged as designer materials that support coupled chromophores with programmable excitonic states and spatial organization that can be integrated into complex structures. ${ }^{37}\left[\frac{47}{4}\right.$ The high fidelity of base pairing ensures that a given DNA design will form predictable nanoscale architectures, and sequencespecific conjugation of chromophores allows for precise positioning within the structure $41+44,48+51$ DNAbased platforms have been extensively used to produce and characterize chromophore dimers. The electronic structure of these dimers has been well established $39[40[52$, and even used to investigate the properties of the DNA itself. ${ }^{38,53}$ They have also been used as a tool to investigate both electronic coherence ${ }^{54}$ and vibronic coherence and its role in the dynamics of excitons within the dimer ${ }^{55}$ While insightful, these studies were limited to single dimers. More recently, a self-assembled chromophore-DNA platform with longer aggregates was developed and used to build higher order structures, which enabled investigation of long-distance exciton transport ${ }^{4456}$ However, in this platform the chromophores were not covalently bound and so lacked discrete control over the aggregate length. Furthermore, none of the previous approaches investigated the 
role of system-bath coupling in exciton transport.

Here, we report the development of chromophore-DNA constructs with control over both electronic coupling and system-bath coupling. We synthesized DNA strands with covalently-attached, sequential indocarbocyanine Cy3 chromophores that we used to fabricate DNA duplexes and higher-order structures with chromophores positioned at desired spatial locations. We characterized the chromophore-DNA constructs with two-dimensional (2D) electronic spectroscopy, single-molecule spectroscopy, and computational modeling. With these constructs, we demonstrated both systematic variation in electronic coupling and aggregate lengths and also scaffold-dependent system-bath coupling. Upon increasing the rigidity of the DNA scaffold, the efficiency of energy transfer to an acceptor chromophore decreased, experimentally demonstrating the way in which the bath can tune the steps underlying long-distance exciton transport. The ability of our chromophore-DNA constructs to simultaneously yet independently change electronic coupling and systembath coupling offers a platform for the design of excitonic systems. These bespoke excitonic systems and their integration into higher order DNA nanostructures lays the groundwork for an emerging material with applications ranging from artificial photosynthesis to quantum sensing and computing.

\section{Results and Discussion}

Controlling exciton formation in DNA scaffolds. Excitons supported by chromophore monomers, dimers or trimers are positioned at desired spatial locations within the DNA scaffold. We inserted one, two, or three consecutive $\mathrm{Cy} 3$ units into the same single-stranded DNA backbone using solid-phase phosphoramidite chemistry and then hybridized the modified strands with complementary single-stranded canonical DNA (Figure 117). In previous DNA-based excitonic systems, chromophore dimers were formed upon annealing of the DNA strands $\frac{37-3957}{57}$ Our synthetic approach of inserting chromophore aggregates into single strands offers three major advantages over other chromophore-DNA systems. First, the aggregate length can be extended beyond dimers in a discrete and controllable fashion, as illustrated here for trimers. Second, the aggregate, or even multiple aggregates, can be modularly positioned within both DNA duplexes and higherorder DNA nanostructures without additional chemical modifications on the complementary strands. It is this ability that enables these constructs to support long-distance exciton transport. Third, there is no monomer contamination due to unannealed single-stranded DNA, which can be present at up to $10 \%$ in 
previous DNA-based excitonic systems $\sqrt[55]{55}$ For initial characterization, we focus on chromophore aggregates within duplexes (Supplementary Information Table S1).

The steady-state absorbance spectra of the chromophore-DNA constructs indicate the formation of aggregates (Figure 1b). The linear absorption spectrum of the monomer features a peak at $18150 \mathrm{~cm}^{-1}$ and a pronounced vibronic progression from strong coupling between the $S_{0} \rightarrow S_{1}$ electronic transition and a C-C stretching mode $\left[\frac{58}{6}\right.$ The linear absorption spectra of the dimer and trimer show markedly different spectral features from the monomer, including a hypsochromic shift of the $0-0$ vibronic peak from $18150 \mathrm{~cm}^{-1}$ for the monomer to $18350 \mathrm{~cm}^{-1}$ for the dimer and the trimer. The hypsochromic shift is consistent with strong electronic coupling between chromophores stacked co-facially, a so-called H-like organization. ${ }^{59}$ The oscillator strength also redistributes from the $0-0$ vibronic band to higher energy vibronic sidebands, namely $0-1$ and $0-2$. Finally, the trimer featured further reduction in the overall oscillator strength of the $0-0$ vibronic band and 0-1 vibronic band. Further spectral analysis is shown in Supplementary Information Section S1.4. The linear absorption spectra were simulated using a vibronic exciton model (Supplementary Information Section S5.7). In order to reproduce the experimentally-measured spectra, a multi-state model was required for the dimer and trimer constructs, suggesting heterogeneity in the aggregate structures (Supplementary Information Sections $\mathbf{S 5 . 8}$ and S5.9.

Fluorescence quantum yields and excited-state lifetimes are known to differ for chromophore aggregates compared to the monomer, providing additional photophysical quantities to establish aggregate formation. $\frac{59}{50}$ The fluorescence quantum yield of the Cy3-DNA duplex constructs decreased with increasing number of Cy3 subunits starting from $24 \pm 1.6 \%$ (mean \pm s.d., $\mathrm{n}=3$ ) for the monomer to $8.9 \pm 0.3 \%$ for the dimer and $3.4 \pm 0.2 \%$ for the trimer. The average fluorescence lifetime of the Cy3-DNA constructs decreased with aggregate length on the DNA, starting from $1.15 \pm 0.21 \mathrm{~ns}$ for the monomer to $0.72 \pm 0.04 \mathrm{~ns}$ and $0.67 \pm 0.01$ ns for the dimer and trimer, respectively (Supplementary Information Section S1.5 and Figure ??). The decrease in fluorescence lifetimes of the Cy3-DNA duplex constructs may be due to enhanced internal conversion rates, which leads to a decrease in quantum yield. An enhancement of internal conversion rates as the aggregate length increases has been observed in other molecular systems.

The fluorescence lifetime data exhibited multi-exponential decay kinetics, which generally reflects het- 


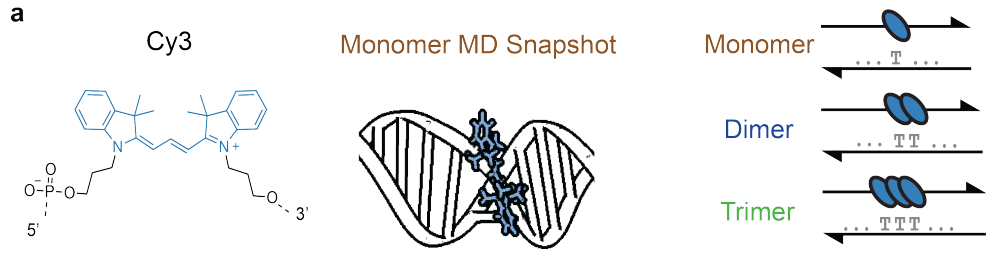

b

C
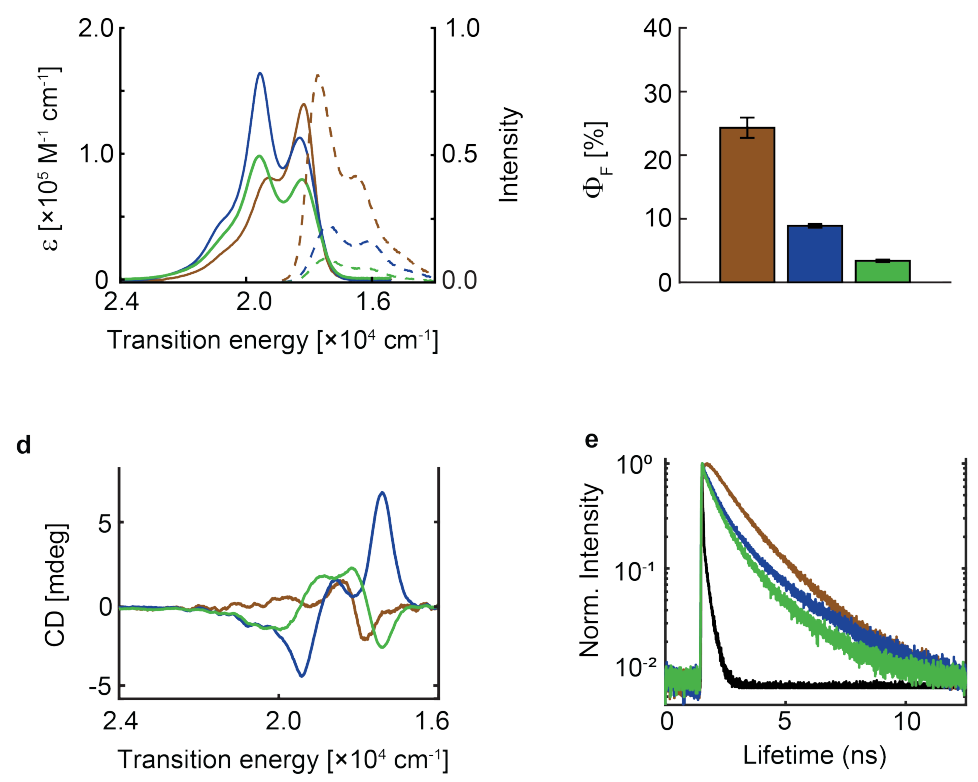

Figure 1 | A chemical approach to form Cy3 aggregate constructs using DNA scaffolds. (a) Cy3 (left) is covalently linked to $3^{\prime}$ and $5^{\prime}$ ends of the deoxyribose-phosphate backbone of single-stranded DNA (ssDNA). Cy3-modified DNA nanostructures are formed by hybridizing Cy3-modified ssDNA with canonical complementary ssDNA strands, as shown in a molecular dynamics snapshot of a Cy3 monomer attached to a DNA duplex (center) and in the schematic (right, top) where blue ovals denote Cy3. Cy3 dimers and trimers are formed by linking consecutive $\mathrm{Cy} 3$ chromophores into ssDNA and hybridizing with complementary strands (right, middle and bottom) (b) Absorbance (solid lines) and quantum yield-normalized fluorescence spectra (dashed lines) of Cy3 monomer (brown), dimer (blue), and trimer (green). [DNA duplex] $=0.5 \mu \mathrm{M}$ in $40 \mathrm{mM}$ Tris, $20 \mathrm{mM}$ acetate, $2 \mathrm{mM}$ ethylenediaminetetracarboxylic acid (EDTA), and $12 \mathrm{mM} \mathrm{MgCl}_{2}$ (TAE- $\mathrm{MgCl}_{2}$ buffer). (c) Fluorescence quantum yields $\left(\Phi_{F}\right)$ of Cy3 monomer, dimer, and trimer in duplexes. [DNA duplex] $=0.5 \mu \mathrm{M}$ in $1 \times \mathrm{TAE}-\mathrm{MgCl}_{2}$ buffer. (d) Circular dichroism (CD) spectra of Cy3 monomers, dimers, and trimers. (e) Fluorescence decay traces of $\mathrm{Cy} 3$ monomers, dimers, and trimers, instrument response function is shown in black. 
erogeneity due to sub-populations within a measured ensemble. The fluorescence lifetime data of the monomeric Cy3-DNA duplex constructs required a bi-exponential function while those of the dimeric and trimeric Cy3-DNA duplex constructs required triexponential functions for an adequate fit, which further suggest heterogeneity in the electronic structure regardless of aggregate length (Supplementary Information Table S3, Figure 17). The existence of heterogeneity was supported by all-atom molecular dynamics (MD) simulations of the monomer (Figure S31). Specifically, the simulations show that the Cy3 monomer can be bound or unbound from the DNA duplex, which leads to a heterogeneous environment for the chromophore ${ }^{63}$ The simulations of the dimer and trimer revealed cofacial stacking, which is also referred to as an H-like aggregate structure (Supplementary Information Section 55.8 , Figures $[\mathrm{S} 32-\mathrm{S} 33$ ). Similar to the case of the monomer, these simulations revealed two major structural states, a weakly coupled, monomer-like state and a strongly coupled dimer state, which lead to localized and delocalized excited-state wavefunctions, respectively.

Controlling electronic coupling within Cy3 dimers in DNA duplexes. We systematically vary the electronic coupling within the $\mathrm{Cy} 3$ dimer constructs by changing the distance and relative orientation between the two chromophores (Figure 2a, Supplementary Information Section S1.7, Table S7). We inserted one to three nucleotide spacers between the $\mathrm{Cy} 3$, where each nucleotide spacer introduces a spatial distance of $\sim 0.34 \mathrm{~nm}$ and $\mathrm{a} \sim 36^{\circ}$ twist angle, as shown in snapshots from molecular dynamics simulations (Figure 2a) ${ }^{64}$ The decrease in electronic coupling with number of inserted nucleotide spacers between the Cy3 dimers is reflected in the steady-state linear absorbance spectra. The hypsochromic shift $\left(190 \mathrm{~cm}^{-1}\right)$, consistent with a coupled dimer in an H-like configuration, is observed across the series. As discussed above, the dimer without nucleotide spacers shows a dramatic redistribution of oscillator strength from the $0-0$ to the $0-1$ band, which is another signature of $\mathrm{H}$-like dimer formation. The redistribution of oscillator strength decreases as the number of nucleotide spacers increases, reflecting the loss of electronic coupling within the dimer due to the increase in intramolecular spacing and changing dipole angle (Supplementary Information Figure S46. When the Cy3 dimers were separated by three nucleotide spacers, the oscillator strength of the $0-0$ band was recovered near the level of the Cy3 monomer. Further, both the fluorescence quantum yield (Figure 25) and fluorescence lifetime approached the monomer values as the number of nucleotide spacers increased (Figure S10). The spectra of the dimers with varied nucleotide spacing can be reproduced 
using a multi-configurational model based on all-atom MD simulations (Figures S47, S49, S51). The multistate configurations identified include strongly coupled, H-like dimers for zero and one nucleotide spacings, while both slip-stacked (known as J-like) and H-like dimers were observed for two nucleotide spacings. $\frac{59}{5}$ Sub-populations of weakly-coupled, monomer-like dimers were also observed for these nucleotide spacings (Supplementary Information Figure S46). Only monomer-like dimers were observed for three nucleotide spacings. The configurational variation shows that, along with control over the magnitude of electronic coupling, these Cy3-DNA constructs offer the ability to select the nature of the aggregate formed.

a

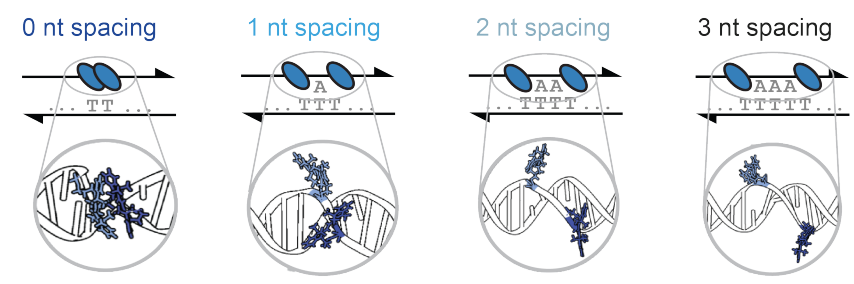

b
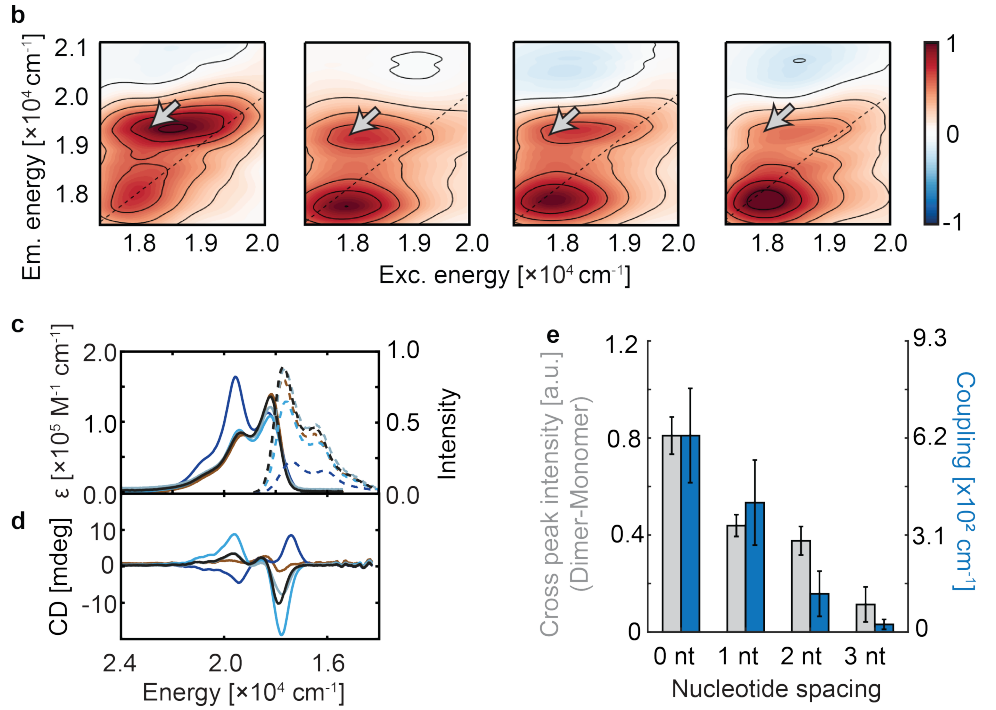

Figure $2 \mid$ Engineering electronic coupling within Cy3 dimers in DNA. (a) The insertion of spacer nucleotides (nt) within dimers increases the distance and changes the relative orientations of the $\mathrm{Cy} 3$ (blue ovals). (b) Representative two-dimensional spectra (250-fs time delay) of each Cy3 dimer for the nt spacing shown above in (a). (c) Absorbance spectra (solid lines) and quantum yield-normalized fluorescence spectra (dashed lines) of Cy3 dimers with varied nt spacings between each Cy3 molecule on the DNA duplex. Absorbance and emission spectra of monomeric Cy3 in the DNA duplex are re-plotted for comparison (brown). [DNA duplex] $=0.5 \mu \mathrm{M}$ in $1 \times \mathrm{TAE}-\mathrm{MgCl}_{2}$ buffer. (d) Circular dichroism spectra of nucleotide spaced dimers (monomer also shown in brown). (e) Comparison of relative upper diagonal cross peak intensity (marked in $\mathbf{b}$ with a grey arrow, scaled by the diagonal peak for the 0-0 transition) from two-dimensional spectra and electronic coupling estimated using transition density cube calculations on molecular dynamics structures.

To experimentally characterize the intra-dimer electronic coupling, we use two-dimensional (2D) elec- 
tronic spectroscopy. 2D electronic spectroscopy measures the excitation frequency-emission frequency correlation and so the signal intensity at the frequencies of two excitonic states increases with the electronic coupling between them. ${ }^{65}$ As shown in Figure $2 b$, the relative coupling between constituent monomer units appears as a cross peak at excitation frequency $\omega_{\mathrm{ex}}=18100 \mathrm{~cm}^{-1}$ and emission frequency $\omega_{\mathrm{em}}=$ $19500 \mathrm{~cm}^{-1}$, which are the energies of the two excitonic states with the most oscillator strength within the dimer. As shown in Figure 2 e, we extracted the upper cross peak intensity to compare the relative coupling between the dimers without contamination from population dynamics or excited state absorption, which contributes to the spectral features in this region (further details are given in Supplementary Information Section S2.2) ${ }^{6970}$ We computed the magnitudes of the electronic coupling using transition density cube calculations (Supplementary Information Section S5.8). The scaling of the experimental and computational results are in good agreement, including strong coupling within the zero nucleotide-spaced dimers, including strong coupling within the zero nucleotide-spaced dimers, and progressively weaker coupling for the 1-3 nucleotide-spaced dimers. However, an underestimation of the relative couplings for the weakly coupled dimers is observed, consistent with the minor discrepancies between the experimental and simulated absorption spectra $[\mathrm{S} 5.9]$, and may be due to variation in the vibronic coupling for different DNA constructs. Overall, the qualitative agreement highlights that our approach includes computational prediction for the design of electronically-coupled constructs.

Dependence of ultrafast exciton-bath coupling on DNA scaffold. Synthetic DNA enables facile integration of chromophores into a variety of DNA scaffolds. ${ }^{71+77}$ Because of our synthetic strategy, Cy3 chromophores can be readily incorporated into a wide range of nanostructures using the same Cy3-modified ssDNA strands, which permits the investigation of scaffold-dependent properties such as system-bath coupling. The double-crossover (DX) tile motif is an essential building block for DNA nanotechnology. DX tiles are at least twice as rigid as duplexes due to inter-duplex crosslinking that couples bending, twisting, and stretching modes $\frac{78,79}{70}$ To investigate scaffold-dependent behavior, we first compared the ultrafast system-bath coupling of free $\mathrm{Cy} 3, \mathrm{Cy} 3$ monomer in duplexes, and $\mathrm{Cy} 3$ monomer in DX tiles (sequences shown in Supplementary Information Section S2.3.

System-bath coupling gives rise to fluctuations in the frequency of a transition, also known as spectral 


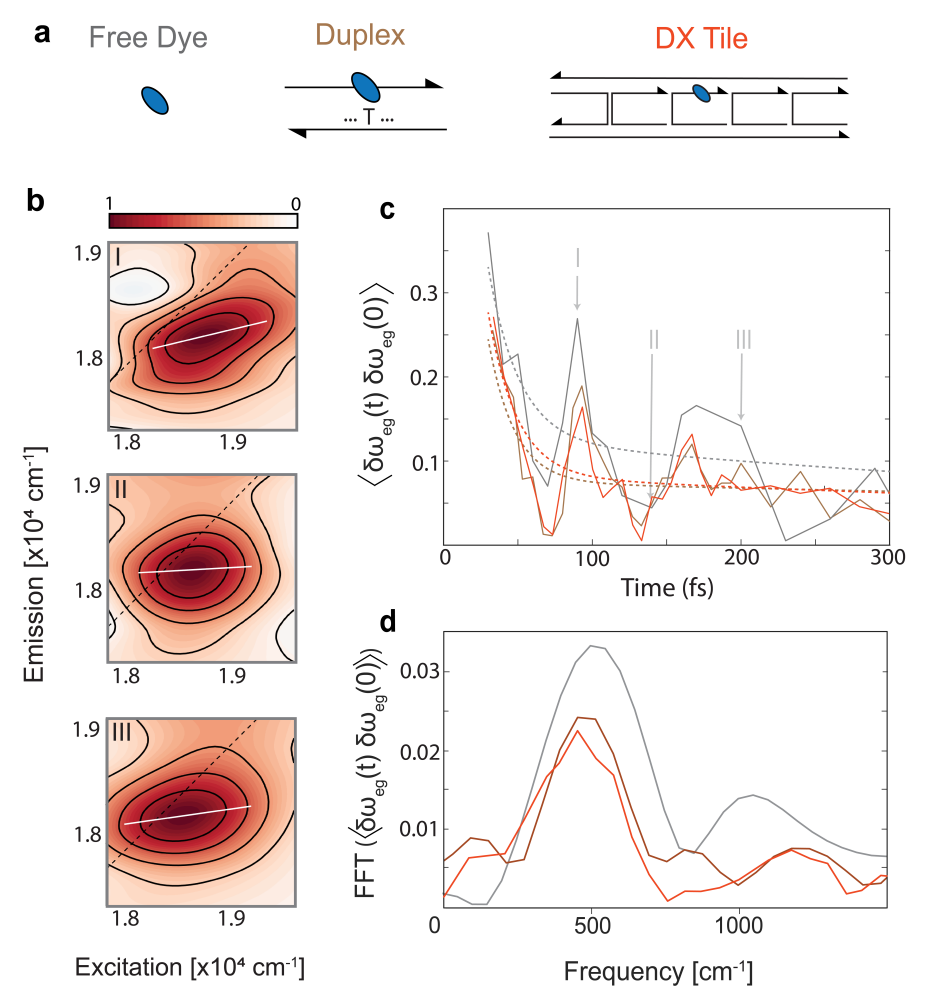

Figure 3 Ultrafast system-bath coupling for monomeric Cy3. (a) Schematic of Cy3 (blue oval) as a free chromophore, in a duplex, and in a DX tile (b) Selected 2D spectra of the free chromophore at varying waiting times (I, II, III indicated in c) with center-line slope (CLS) of the 0-0 transition shown in white. (c) Frequency-frequency correlation function derived from the CLS (solid lines) for $\mathrm{Cy} 3$ as a free chromophore (gray), in a duplex (brown) and in a DX tile (red) with fit of exponential decay (dotted lines). (d) Power spectrum of frequency-frequency correlation function (50-200 fs) for all three samples. All samples were measured at a concentration of $100 \mu \mathrm{M}$ in $1 \times$ TAE- $\mathrm{MgCl}_{2}$.

diffusion, on a range of timescales. The frequency-frequency correlation function describes the timescale of the fluctuations. On the ultrafast timescale, the overall decay in the correlation function arises from dephasing due to coupling of the electronic transition to a near-continuum set of modes in the bath, known as overdamped modes. The oscillations in the correlation function arise from coupling to underdamped modes in the bath, generally normal modes of the chromophore. Because 2D spectroscopy probes the excitation frequency-emission frequency correlation, the frequency-frequency correlation function is contained in the temporal dynamics of the spectra. The temporal dynamics are measured by recording a series of $2 \mathrm{D}$ spectra as a function of a delay time between excitation and emission events. The frequency-frequency correlation for a given electronic transition is proportional to the center-line slope of its peak in the 2D spectra (Figure 33$) .80-83$ The center-line slope for the series of delay times is used to construct the frequency-frequency correlation function. 
We compare the dynamics of the frequency-frequency correlation for the lowest energy transition, the 0-0 band, for monomeric Cy3 under all three scaffold conditions (Figure 3 ). The correlation function decays rapidly for all samples with average timescales of $290 \pm 40 \mathrm{fs}, 120 \pm 40 \mathrm{fs}$, and $100 \pm 40$ fs for the free chromophore, the duplex, and the DX tile, respectively (biexponential fit parameters are shown in Supplementary Information Section S2.5). The femtosecond decay constant is due to fast spectral diffusion, similar to previously studied chromophores in solution ${ }^{84}$ (85 The correlation function oscillates for up to 200 fs for all samples, reflecting the presence of a strongly coupled underdamped vibrational mode. The Fourier transform of the decay-subtracted correlation function for delay times 50-200 fs is shown in Figure $3 \mathrm{~d}$. The frequency-domain data has a broad peak centered at $450 \mathrm{~cm}^{-1}$, which suggests coupling to approximately the same underdamped modes regardless of scaffold. In addition, homodyne transient grating measurements show coupling to approximately the same modes through the presence of a peak at the same frequency (Figure S17 ) $^{86}$. As illustrated in Figure S17b, the broad peak observed in Figure $3 \mathrm{~d}$ is consistent with rapid dephasing of normal mode vibrations delocalized over Cy3 from $250-750 \mathrm{~cm}^{-1}$. The free Cy3 in solution showed larger amplitude oscillations than the DNA-bound Cy3 (Supplementary Information Section S2.5). This indicates that covalent attachment to DNA reduces the degree to which the electronic transition dipole couples to the underdamped bath mode or modes. The short timescale decays and amplitude oscillations observed for both the duplex and DX tile reflect their similar local environments on the nanometer length scale, as opposed to the variation in persistence length on the tens of nanometers scale 79 . The faster decorrelation may arises due to the charge intrinsic to DNA, including the negatively charged backbone and associated counter ions, which is constant across the varying constructs and could cause even faster charge redistribution upon photoexcitation of the positively charged dye, thus driving rapid fluctuations in the transition energy.

Impact of DNA scaffold on heterogeneity. Heterogeneity between individual Cy3-DNA constructs, also known as static disorder, arises from the coupling of slow, anharmonic motions of the DNA scaffold to the electronic transitions. This long timescale system-bath coupling gives rise to differences in the transition frequency and other photophysical parameters of the constructs. To initially investigate the impact of the DNA scaffold on heterogeneity between constructs, the fluorescence lifetimes of $\mathrm{Cy} 3$ in duplexes and in DX tiles were compared, as the components of a multiexponential lifetime decay generally correspond to 
subpopulations, as discussed above. At the ensemble level, the measured fluorescence lifetimes were similar for $\mathrm{Cy} 3$ in the two scaffolds. Fits to the fluorescence lifetime required a bi-exponential decay for monomers and a tri-exponential decay for dimers and trimers, reflecting at least two and at least three subpopulations, respectively (Supplementary Information Section S1.5.

To further investigate heterogeneity between Cy3-DNA constructs, the fluorescence lifetimes of individual constructs were measured using single-molecule fluorescence spectroscopy. These measurements benchmark the distributions of photophysical properties for $\mathrm{Cy} 3$ monomers and dimers in DNA as the widths of the distributions directly report on the heterogeneity of the excitonic states. Single-molecule measurements were performed on monomeric and dimeric $\mathrm{Cy} 3$ in duplexes and $\mathrm{Cy} 3$ in DX tiles. Each construct required a bi-exponential decay function for a good fit, reflecting the presence of at least two subpopulations in each construct that interconvert on a timescale longer than the nanosecond lifetime but shorter than the tens of milliseconds required to detect sufficient emission to construct a decay curve (Supplementary Information Section S4. The shortest component from the ensemble dimer decay was unobserved, likely because it was hidden in the longer instrument response function of the single-molecule apparatus.

To investigate the distribution of lifetimes, the average lifetimes for each construct were used to build a histogram for each sample (Figure $4 \mathrm{~b}$ and c). All of the resultant distributions showed one primary peak, although the widths varied, indicating different levels of heterogeneity. For Cy3 monomers, the full-width half maximum in a duplex $(0.90 \mathrm{~ns})$ is more than four times broader than the full-width half maximum in a DX tile $(0.20 \mathrm{~ns})$. Consistently, for Cy3 dimers, the full-width half maximum in a duplex (0.60 ns) is broader than in a DX tile (0.30). These differences in heterogeneity arise from differences in the systembath coupling due to the properties of the DNA scaffold. The scaffold design is, therefore, a tool to control the system-bath coupling and thereby tune the heterogeneity of the excitons themselves.

To investigate the structural differences responsible for the lifetime differences between the two scaffolds, MD simulations were performed for monomeric and dimeric Cy3 in duplexes and in DX tiles. Solvent accessible surface area (SASA) of $\mathrm{Cy} 3$ in each construct was extracted from the simulation data (Figure $4 \mathrm{~d}$ and e). The SASA distributions are broader for both monomeric and dimeric Cy3 in duplexes compared to the DX tile counterparts. The SASA distribution for monomeric Cy3 in duplexes is bimodal, showing 


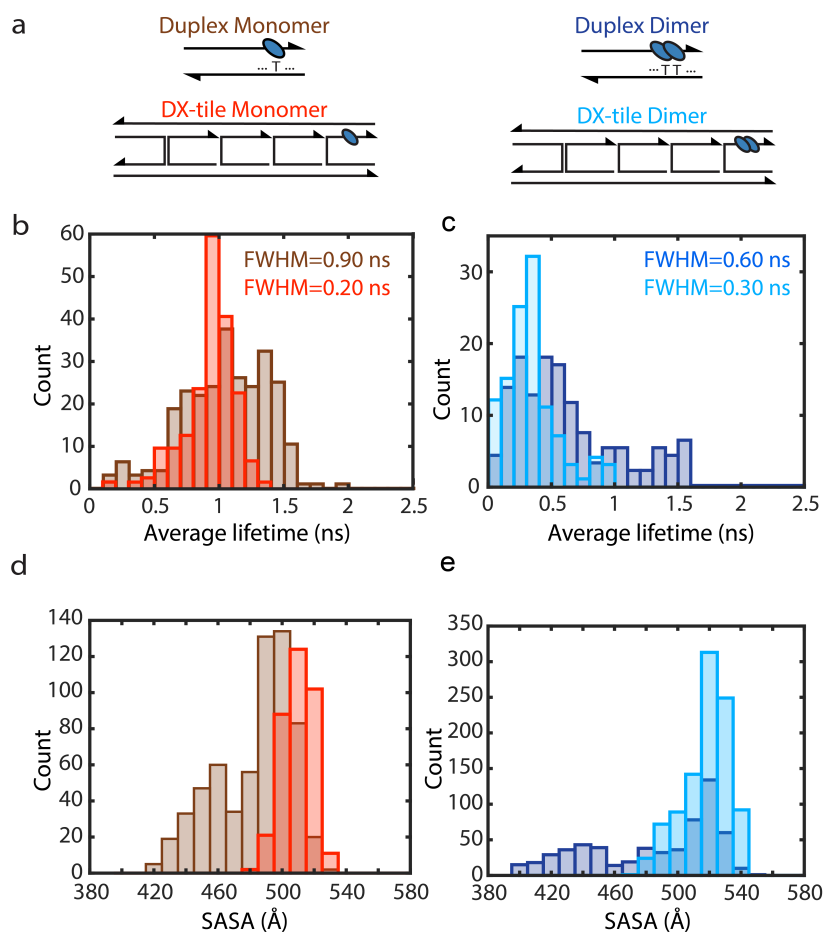

Figure $4 \mid$ Heterogeneity of Cy3 in DNA scaffolds. (a) Schematics of monomeric (left) and dimeric (right) Cy3 in duplexes (top) and DX tiles (bottom). (b) Histograms constructed from the average lifetimes of individual constructs for monomeric Cy3 in duplexes (brown) and in DX tiles (red) measured with single-molecule spectroscopy. (c) Histograms constructed from the average lifetimes of individual constructs for dimeric Cy3 in duplexes (dark blue) and in DX tiles (bright blue) measured with single-molecule spectroscopy. (d) Histograms constructed from the MD trajectory of solvent exposed surface area (SASA) for monomeric Cy3 in duplexes (brown) and in DX tiles (red). (e) Histograms of SASA for dimeric Cy3 in duplexes (dark blue) and in DX tiles (bright blue).

two populations centered at $460 \AA$ and at $500 \AA$ whereas the distribution for monomeric Cy3 in DX tiles is unimodal and narrow, centered at $510 \AA$. Similarly, the SASA distribution of dimeric Cy3 in duplexes is bimodal, with populations centered at $440 \AA$ and at $520 \AA$, whereas the distribution for dimer Cy3 in DX tiles is unimodal, centered at $520 \AA$. The differences in width and modality likely originate from the differences in rigidity between the two scaffolds (Figure S43). The DX tile rigidity restricts kinking of the structure whereas the duplex flexibility allows kinking (bending), leading to conformations in which the chromophore is embedded in the DNA groove, where the SASA is low ${ }^{7987}$ The lifetime of Cy3 has been shown to depend strongly on the solvent environment, which affects the rates of excited-state processes such as internal conversion pathways and photoinduced isomerization. $[0]$ Therefore, the broader SASA distribution is likely the cause of the broader distribution of lifetimes for the duplex-scaffolded monomer and dimer as compared to the DX-scaffolded chromophores. 
While system-bath coupling is often studied in the limiting regimes of static disorder and ultrafast dynamics, fluctuations of the excitonic system occur on many timescales. Motions of the DNA scaffold also gives rise to structural fluctuations of the $\mathrm{Cy} 3$ chromophore on the timescale of energy transfer. For the Cy3 monomer, depolarization of the transition dipole moment is due to motion of the chromophore and can be measured with ensemble time-resolved fluorescence anisotropy. The anisotropy is calculated from separately-detected parallel and perpendicular components of the fluorescence emission and is shown in Figure 5 a for monomeric Cy3 in duplexes and in DX tiles. The anisotropy decay curves were fit with a two timescale model. The short-time constants for the monomer in duplexes and in DX tiles were $0.19 \pm 0.15 \mathrm{~ns}$ and $0.62 \pm 0.14 \mathrm{~ns}$, respectively, while the long-time constants were $3.45 \pm 0.94 \mathrm{~ns}$ and $22.97 \pm 16.22 \mathrm{~ns}$, respectively (Table S18). The long-time constant was assigned to the tumbling of the DNA construct, which is faster for the duplex due to its smaller size, and corresponding higher rotational diffusivity, relative to the DX tile. The short-time constant was assigned to local structural fluctuations of the chromophore. The amplitude of the short-time constant can be related to an order parameter from which a cone describing the extent of local fluctuations can be calculated. ${ }^{89}$ Using this model, the difference in half-cone angle between Cy3 monomers in duplexes and in DX tiles is $\Delta \theta=8.8 \pm 4.6^{\circ}$ (Supplementary Information Section S3.5), where Cy3 monomers on DX tiles were found to have more local freedom than Cy3 monomers on duplexes. The increase in local freedom is likely due to the difference in kinking, as discussed above. In the MD simulations, the duplex kinks towards the Cy3 (Supplementary Figure S40), confining the chromophore and restricting its motion.

Dependence of energy transfer efficiency on DNA scaffold. To investigate exciton transport with the Cy3-DNA constructs, we attached a Cy5 monomer with a deoxythymidine spacer to the terminal end of the DNA to serve as an acceptor (Figure 5b). The efficiency of energy transfer from the Cy3 monomer or dimer to the Cy5 acceptor was quantified by ensemble steady-state fluorescence measurements (Figure $5 \mathrm{~d}$, Supplementary Information Section S3.3). An energy-transfer efficiency of $95 \%$ was found for monomeric $\mathrm{Cy} 3$ in duplexes while the efficiency decreased to $90 \%$ for dimeric Cy3 in duplexes, which can be attributed to the reduced quantum yield of the dimer relative to the monomer (Figure 11). In contrast, an efficiency of $75 \%$ was found for Cy3 monomers in DX tiles and of $70 \%$ for Cy3 dimers in DX tiles (Supplementary Information Sections S3.1 S3.4. A 20\% drop in energy-transfer efficiency was observed for Cy3 in DX 
tiles compared to in duplexes, even though the number of nucleotides between the donor and acceptor was maintained in both scaffolds.

To understand the molecular origin of the scaffold-dependent efficiency, we individually examine the photophysical quantities involved in energy transfer. The Cy3 monomer or dimer and the Cy5 are $\sim 2.7$ $\mathrm{nm}$ apart, and at this distance energy transfer occurs through Förster resonance energy transfer (FRET). The efficiency of FRET depends on the quantum yield of the donor chromophore, the extinction coefficient of the acceptor, the transition energies of the chromophores, the distance between them, and the angle between their transition dipole moments. ${ }^{90 \mid 94}$ The quantum yields, molar absorption coefficients, and transition energies are the same for both the duplexes and DX tiles (Supplementary Information Figure S15 and Figure S22). The distances are similar as the same local nucleotide sequence was used (Supplementary Information Sections S3.1) and the MD simulations show little scaffold-specific displacement of the chromophore (Supplementary Information Section S5.6). Therefore, the angle between the transition dipole moments is the likely origin of the difference.

The FRET rate is sensitive to thermal fluctuations in the orientations of the transition dipole moments and thus the ensemble FRET efficiency depends on the angular distribution that these fluctuations generate .95 Both the timescale of the fluctuations and the shape of the distribution can influence the overall energy transfer efficiency. In the case where fluctuations are fast relative to energy transfer time scales, transition dipoles sample a large number of different configurations between energy transfer events. In the dynamic limit, ensemble FRET efficiency is determined by a single mean energy transfer rate, given by the average over the angular distribution. In contrast, when fluctuations are slow relative to energy transfer timescales, transition dipoles sample very few configurations between energy transfer events. In the static limit, the distribution of rates becomes a distribution of efficiencies, the latter of which is averaged to determine the ensemble FRET efficiency.

The fluctuations in transition dipole orientation are often assumed to be normally distributed with a variance determined experimentally using fluorescence anisotropy. This assumption is valid in the dynamic limit because a normal distribution is sufficient to capture the average behavior. In the chromophore-DNA systems, the normal distribution is visualized as a cone centered along the transition dipole moment of the 

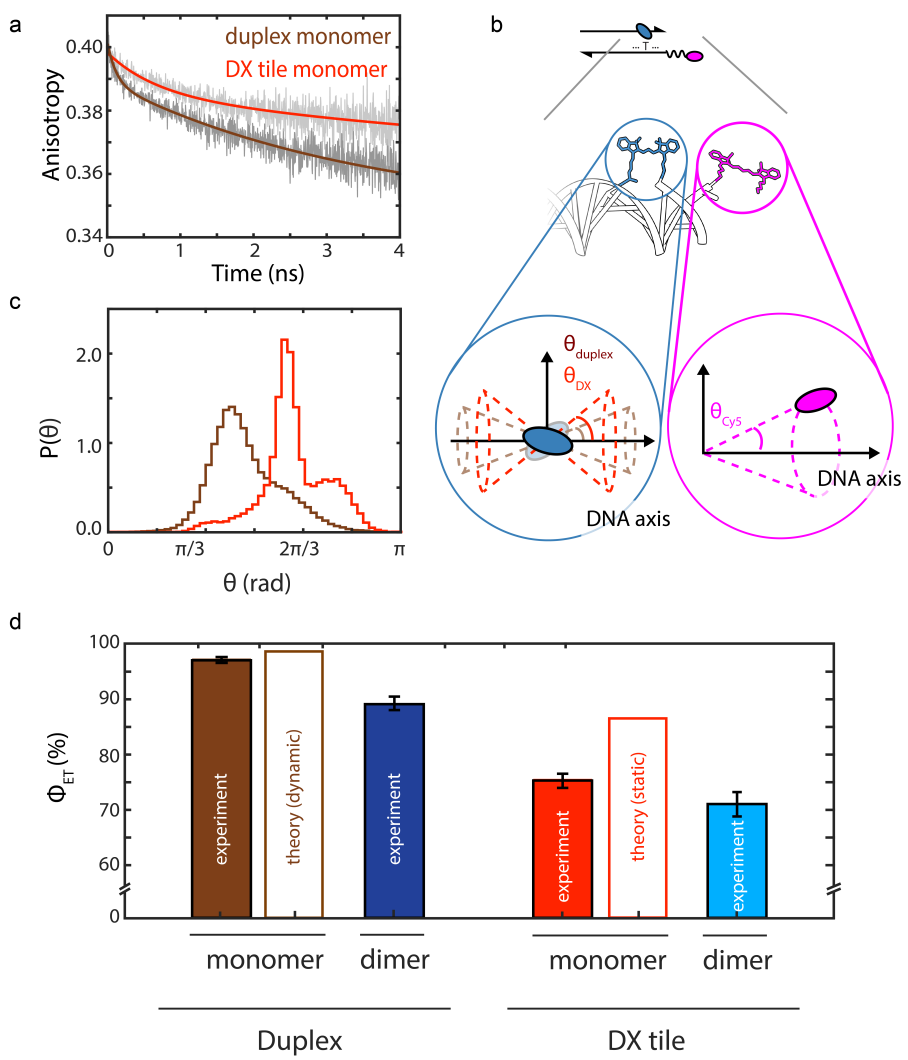

Figure 5 Effect of DNA scaffold on energy transfer efficiency. (a) Fluorescence anisotropy decay curves for monomeric Cy3 in duplexes (data, light gray; fit, brown) and DX tiles (data, dark gray; fit, red). Fit parameters are in Table S18 (b) Schematic of monomeric Cy3 in DNA with acceptor (top). The cone angles extracted from the anisotropy decays and their expected orientation relative to the DNA axis are illustrated for Cy3 in duplexes and DX tiles (bottom left) and for Cy5 (right). (c) The angle between the transition dipole moment of monomeric Cy3 in DX tile monomers and the DNA backbone obtained from MD simulations. (d) Energy transfer efficiency from ensemble fluorescence measurements (solid) and from FRET calculations (stripes) for monomeric and dimeric Cy3 in duplexes and DX tiles. The efficiency is $\sim 20 \%$ lower in the DX tiles than in the duplexes.

chromophore, which is at a position and orientation determined by the geometric constraints imposed by the linkers, as illustrated in Figure $5 \mathrm{~b} \cdot \frac{9596}{96}$ The cone angle of Cy5 is $\theta_{\mathrm{Cy} 5}=33^{\circ} \pm 2^{\circ}$ centered on the axis parallel to the DNA backbone, as illustrated in Figure $5 \mathrm{~b}^{96}$ (Supplementary Information Section S3.5). The cone angle of $\mathrm{Cy} 3$ is centered parallel to that of $\mathrm{Cy} 5$ and, as discussed above, is narrower for monomeric Cy3 in duplexes than in DX-tiles (Supplementary Information Section S3.5). The efficiencies calculated with the normal distribution model are $98 \%$ and $97 \%$ for the duplex and DX tile, respectively (Supplementary Information Table S19. While the efficiency for the duplex is close to the experimentally-measured value, the efficiency for the DX tile is significantly over-estimated (Figure $5 \mathrm{~d}$ ). The anisotropy data revealed that the fluctuations for $\mathrm{Cy} 3$ on duplexes are $\sim 3-4$ times faster than for $\mathrm{Cy} 3$ on DX tiles, suggesting that the 
dynamic limit may not be appropriate for the DX tile.

In the static limit, the shape of the angular distribution can have a significant effect on FRET efficiency, so the approximation of a normal distribution is not necessarily reliable. In fact, the efficiencies calculated in the static limit with the normal distribution model are nearly identical to those calculated in the dynamic limit, $98 \%$ and $97 \%$ for the duplex and DX tile, respectively. To evaluate the validity of the normal distribution, we extracted the distributions from the all-atom MD simulations of $\mathrm{Cy} 3$ on both scaffolds, which exhibit a structured profile with differences between the two constructs (Figure 5k). This structure arises due to the constraints of the two-point tether and steric interactions between Cy3 and the DNA scaffold that cause $\mathrm{Cy} 3$ to rotate out of alignment with the DNA backbone. For the DX tile distribution, the FRET efficiency in the static limit is $86 \%$, which is closer to the experimental value than the normal model, and illustrates that the nature of the distribution can exert a significant influence on the overall energy transfer efficiency in the static limit. For the duplex distribution, the FRET efficiency is $83 \%$ in the static limit, which underestimates the experimental value. The corresponding efficiency in the dynamic limit is $98 \%$, consistent with the experimental value. These results suggest that the static limit is more appropriate for the DX tile whereas the dynamic limit is more appropriate for the duplex, as expected based on the timescales of the fluctuations from the anisotropy measurements, and it is this difference in timescales that is the source of the difference in the FRET efficiency.

While the effect of the average angle between the transition dipole moments is well known 92 9798 these results demonstrate that the nature and timescales of the fluctuations that shape the distribution of angles ultimately determine the measured FRET efficiencies. Although the efficiency is independent of the timescale for the commonly used normal model, some condensed phase systems can have a structured angular distribution that, in the static limit, restricts the overall FRET efficiency, as observed for the DX tile scaffold. The efficiency can be recovered, however, by rapid fluctuations that in the dynamic limit, as observed for the duplex tile scaffold. This picture is in contrast to the typical view of fluctuations as a disruption to a process of interest and provides a mechanism to maintain high efficiency, which may even be playing a role in photosynthetic energy transfer where high quantum efficiency is observed despite the fluctuations of the protein environment. 
Our measurements are an experimental demonstration that the properties of the DNA scaffold itself, i.e., the bath, impact the dynamics of excitonic systems, including transport. While the impact of the bath on exciton dynamics had been well established theoretically 199 - 101 , previous experiments were limited to the impact of the bath on individual excitonic states ${ }^{102}$. Chromophore-DNA constructs open the door to varying the system-bath coupling for chromophore aggregates, and thus achieving the full nanoscale control required to direct exciton dynamics.

\section{Conclusion}

Here, we develop a DNA-based platform for synthetic control over excitonic systems. While the interplay of electronic coupling and system-bath coupling was known to guide exciton transport, previous approaches were unable to simultaneously vary both couplings. We show the importance of this ability by demonstrating bath-dependent energy transfer efficiency. Characterization and manipulation of electronic coupling and system-bath coupling in individual duplexes and DX tiles, the building block of DNA origami, provides a path towards integration of our excitonic system into higher-order objects ${ }^{73}$-77, which is not easily accessible with other synthetic methods 39,40 . The production of higher-order structures provides access to novel materials with prescribed properties and geometric structures for broad ranging applications including light harvesting and information processing.

\section{Methods}

Synthesis procedure for Cy3-modified strands. Cy3-modified oligonucleotides (sequences are listed in the Supplementary Information Table S1, Table S7, Table S9, and Table S13, were prepared via automated oligonucleotide synthesis on a 394-DNA/RNA synthesizer (Applied Biosystems) following previously published procedures $\frac{51}{}$ UltraMild CE phosphoramidites, UltraMild supports, and special Cap Mix A (tetrahydrofuran/pyridine/phenoxyacetic anhydride) were used to synthesize the oligomers on support. Cyanine-3 phosphoramidite, UltraMild CE phosphoramidites, and other reagents for the synthesis of the oligonucleotides were purchased from Glen Research (Sterling, VA) or Sigma-Aldrich (Buchs, Switzerland). The coupling time for nucleotide-to-cyanine, cyanine-to-cyanine, and cyanine-to-nucleotide coupling steps was increased to 3 minutes. For all other nucleotide couplings, the standard coupling time ( 25 seconds) 
was used. Cleavage from the solid support and final deprotection involved treatment with $50 \mathrm{mM}$ potassium carbonate solution in methanol at room temperature for 4 hours. The oligonucleotides were purified by ionpair reversed-phase high-performance liquid chromatography using a Shimadzu LC system equipped with a Shimadzu-block temperature controller (Supplementary Information Section S1.2, Supplementary Figures S1, 33 .

Preparation and purification of DNA constructs. Unmodifed single-stranded DNA were obtained from Integrated DNA Technologies (Coralville, IA) and were received as dry pellets. All strands required to form either DNA duplexes or DX tiles were re-constituted into DNAse/RNAse-free water at $500 \mu \mathrm{M}$ concentrations and kept at $-20^{\circ} \mathrm{C}$ until further use. Equimolar concentrations $(50 \mu \mathrm{M})$ of oligonucleotide strands required to form either duplexes or DX tiles were added into nuclease-free water. A volume of $10 \times$ TAE- $\mathrm{MgCl}_{2}$ buffer (400 mM Tris, $100 \mathrm{mM}$ acetate, $20 \mathrm{mM}$ EDTA, $120 \mathrm{mM} \mathrm{MgCl}_{2}$ ) was added into the mixture to have a final concentration of $1 \times$ TAE- $\mathrm{MgCl}_{2}(40 \mathrm{mM}$ Tris, $10 \mathrm{mM}$ acetate, $2 \mathrm{mM}$ EDTA, $12 \mathrm{mM}$ $\mathrm{MgCl}_{2}$ ). Buffer concentrations for samples used in all experiments were maintained at $1 \times \mathrm{TAE}-\mathrm{MgCl}_{2}$. For duplexes, the mixture was subjected to the following annealing protocol: 2 minutes at $95^{\circ} \mathrm{C}, 80^{\circ} \mathrm{C}-70^{\circ} \mathrm{C}$ at $-2^{\circ} \mathrm{C} \min ^{-1}, 70^{\circ} \mathrm{C}-50^{\circ} \mathrm{C}$ at $-1^{\circ} \mathrm{C} \min ^{-1}, 50^{\circ} \mathrm{C}-20^{\circ} \mathrm{C}$ at $-2^{\circ} \mathrm{C} \min ^{-1}$. An extended annealing protocol was used for DX tiles: 2 minutes at $95^{\circ} \mathrm{C}, 80^{\circ} \mathrm{C}-70^{\circ} \mathrm{C}$ at $-1^{\circ} \mathrm{C} \min ^{-1}, 70^{\circ} \mathrm{C}-50^{\circ} \mathrm{C}$ at $-0.5^{\circ} \mathrm{C} \min ^{-1}$, $50^{\circ} \mathrm{C}-20^{\circ} \mathrm{C}$ at $-1^{\circ} \mathrm{C} \min ^{-1}$ (melting curves shown in Supplementary Information Section S1.3, Figure S4). The annealed mixtures were then purified with native polyacylamide gel electrophoresis (PAGE) using a Biorad Mini-PROTEAN@system that was equipped with 1.5-mm spaced glass plates for gel casting and 10-well, 1.5-mm thick well combs. Annealed DNA duplex samples were loaded into 12\% PAGE gels (400 pmole per well) that were supplemented with $1 \times \mathrm{TAE}-\mathrm{MgCl}_{2}$ buffer. Annealed DX tile samples were loaded into $10 \%$ PAGE gels (400 pmole per well) that were also supplemented with $1 \times \mathrm{TAE}-\mathrm{MgCl}_{2}$ buffer. Gels were ran at $100 \mathrm{~V}$ at constant voltage for 45 minutes at room-temperature. After electrophoresis, the gels were examined through a Vernier BlueView Transilluminator (Beaverton, OR) and the bands of interest were excised. The excised gel pieces were coarsely chopped, collected into a 500- $\mu$ L Eppendorf DNA LoBind microcentrifuge tubes that have pierced small holes ( $\sim 1 \mathrm{~mm}$ diameter) at the pointed end, and finally placed into 2.0-mL Eppendorf DNA LoBind microcentrifuge tube. The microcentrifuge assembly containing the 
excised gel pieces were spun at $15000 \times \mathrm{g}$ for 1 minute. The gel pieces that passed through the hole of the $500-\mu \mathrm{L}$ microcentrifuge tube and into the $2.0-\mathrm{mL}$ tube were pooled together into a $15-\mathrm{mL}$ tube, and $1 \times$ TAE- $\mathrm{MgCl}_{2}$ buffer was added until all the gel pieces were covered with buffer. The mixture was incubated for 14 hours at $35^{\circ} \mathrm{C}$, then vortexed for 10 seconds to re-disperse the gel pieces and transferred into several Biorad Freeze 'N Squeeze ${ }^{\mathrm{TM}}$ tubes, which were spun at $10000 \times \mathrm{g}$ for 1 minute. The supernatant from each

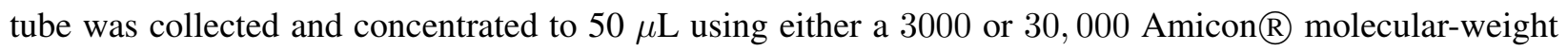
cutoff microcentrifuge filters for duplexes and DX tiles, respectively. Samples were finally stored at $4^{\circ} \mathrm{C}$ in $1 \times$ TAE- $\mathrm{MgCl}_{2}$ for no longer than 1 week.

Steady-state ensemble spectroscopic characterization. Absorbance spectra were measured using an Evolution 260 Bio UV-Vis spectrophotometer (Thermo-Fisher) and fluorescence spectra $\left(\lambda_{\mathrm{exc}}=515 \mathrm{~nm}\right)$ were measured using a FluoroMax-4C (Horiba Jobin Yvon). For fluorescence measurements, a sample absorbance of 0.1 or less was maintained to avoid reabsorption effects. Quantum yields of DNA constructs were determined using the relative quantum yield determination method with rhodamine 101 in spectroscopic-grade ethanol ( $\Phi_{F}=0.92$ measured using integrating sphere method) as reference ${ }^{103}$. All fluorescence spectra are corrected for lamp fluctuations and detector sensitivity (or S1c/R1c detector setting). The absorbance and fluorescence spectra of all the samples were measured in 10-mm pathlength quartz microcuvettes (Millipore Sigma, catalog number: Z802662).

Steady-state ensemble energy-transfer efficiencies were estimated using steady-state fluorescence quenching experiments. The energy-transfer efficiency $\left(\Phi_{\mathrm{ET}}\right)$ of $\mathrm{Cy} 3$ to Cy5 was estimated by

$$
\Phi_{\mathrm{ET}}=1-\frac{\int_{\lambda_{\mathrm{em}, \min }}^{\lambda_{\mathrm{em}, \max }} I_{\mathrm{Cy} 3+\mathrm{Cy} 5} d \lambda_{\mathrm{em}}}{\int_{\lambda_{\mathrm{em}, \min }}^{\lambda_{\mathrm{em}, \max }} I_{\mathrm{Cy} 3} d \lambda_{\mathrm{em}}}
$$

where $\int_{\lambda_{\mathrm{em}, \min }}^{\lambda_{\mathrm{em} \text { max }}} I_{\mathrm{Cy} 3+\mathrm{Cy} 5}$ and $\int_{\lambda_{\mathrm{em}, \text { min }}}^{\lambda_{\mathrm{em} \text { max }}} I_{\mathrm{Cy} 3}$ are the integrated fluorescence intensities of Cy3 measured from $\lambda_{\mathrm{em}, \max }=520 \mathrm{~nm}$ to $\lambda_{\mathrm{em}, \min }=750 \mathrm{~nm}$ in the presence or absence of Cy5, respectively. All samples were 
excited at $\lambda_{\text {exc }}=515 \mathrm{~nm}$. The full fluorescence spectra of Cy3 used to calculate $\int_{\lambda_{\mathrm{em}, \min }}^{\lambda_{\mathrm{em}, \max }} I_{\mathrm{Cy} 3+\mathrm{Cy} 5}$ were obtained by spectral decomposition of the $\mathrm{Cy} 3$ and $\mathrm{Cy} 5$ spectra using least-squares fitting which is detailed in Supplementary Information Section $\mathrm{S3.3}$

Ensemble time-resolved fluorescence lifetime. Ensemble fluorescence lifetime measurements were performed with a pulsed excitation source centered at $520 \mathrm{~nm}$. A supercontinuum was generated by focusing a Ti:Sapphire laser (Vitara-S, Coherent; $\lambda_{c}=800 \mathrm{~nm}, \Delta \lambda=70 \mathrm{~nm}, 20$ fs pulse duration, $80 \mathrm{MHz}$ repetition rate) into a nonlinear photonic crystal fiber (FemtoWhite 800, NKT photonics) and the desired spectral range was selected using a bandpass filter (Thorlabs; FB520-10). Fluorescence was collected at magic angle and passed through a long-pass filter (Omega Optics; 560LP RapidEdge), an inverted long-pass filter (Semrock; BLP01-647R-25) and a bandpass filter (Omega Optics; 580BP100 RapidBand). Single photons were collected by an SPAD (Micro Photon Devices) and arrival times were recorded by a time-correlated single photon module (Picoquant; PicoHarp 300). IRF was measured to be 56 ps. Histogrammed fluorescence lifetime was analyzed by least-squares reconvolution and fitted to the minimum number of components required to obtain the least structured residual.

Fluorescence anisotropy was obtained by time-resolved polarized fluorescence experiments. The excitation and emission filters used were the same as above (Supplementary Information Section S18. Polarized fluorescence decay traces, parallel $\left(I_{\|}(t)\right)$ and perpendicular $\left(I_{\perp}(t)\right)$, were collected and used to calculate time-resolved fluorescence anisotropy, $r(t)$, as follows:

$$
\begin{aligned}
& r(t)=\frac{I_{\|}(t)-G \times I_{\perp}(t)}{I_{\|}(t)+2 \times G \times I_{\perp}(t)} \\
& r(t)=\left(r_{0}-r_{\infty}\right)\left[A \exp \left(-t / \tau_{1}\right)+(1-A) \exp \left(-t / \tau_{2}\right)\right]+r_{\infty}
\end{aligned}
$$

$G$ was determined experimentally to be 0.43 using the intensity ratio of rhodamine 101 solution in 
glycerol:

$$
G=\frac{I_{\mathrm{HV}}}{I_{\mathrm{HH}}}
$$

where $I_{\mathrm{HV}}$ and $I_{\mathrm{HH}}$ are the background-subtracted fluorescence intensities excited with perpendicularly polarized light and detected in parallel and perpendicular, respectively.

Femtosecond spectroscopy. Details of two-dimensional electronic spectroscopy are given in the Supplementary Information and the set-up is described elsewhere. ${ }^{104} 105$ Briefly, $2.8 \mathrm{~W}$ of the $5 \mathrm{~W}$ output from a 5 kHz Ti:sapphire regenerative amplifier (Coherent Libra) with a 40-fs duration and 800-nm center wavelength was focused into a 1-meter tube of argon to generate a broadband continuum. The sub $805 \mathrm{~nm}$ portion of the continuum was filtered with an Schott glass filter (Thorlabs FGV9) to provide a center wavelength of 530 $\mathrm{nm}$ with a FWHM of $50 \mathrm{~nm}$. The pulse was compressed to $11 \mathrm{fs}$ by two pairs of chirped mirrors (Ultrafast Innovations $\mathrm{GmbH}$ ) as characterized by a transient grating frequency resolved optical gating experiment (Supplementary Information Section S2.1). The 2D data were collected in a BOXCARS geometry $67 \mid 104$ with an attenuated local oscillator $\left(\Delta t_{\mathrm{LO}}=500 \mathrm{fs}\right)$. The coherence times were scanned with $0.25 \mathrm{fs}$ steps from -175 fs to $+175 f$ s. Further details are given in Supplementary Information Section $\mathbf{S} 2.1$.

Homodyne transient grating measurements were performed with the same geometry as the 2DES experiments in the absence of a local oscillator. Here, the time delay between pulses one and two was 0 fs while the time delay between pulses 2 and 3 was scanned to recover the transient grating spectrum at time steps of 1.3 fs for delay times from 0 to 2 ps.

Single-molecule fluorescence spectroscopy. Single-molecule fluorescence lifetime measurements were performed with a home-built confocal microscope. Excitation light centered at $550 \mathrm{~nm}$ was obtained by filtering the same supercontinuum generated above for ensemble fluorescence spectroscopy with a bandpass filter (Omega Optics; RPB 540-560 RapidBand). Fluorescence was collected using the same emission filter set as for ensemble time-resolved fluorescence spectroscopy described above. Excitation power was set 
to $5.5 \mu \mathrm{J} / \mathrm{cm}^{2}$ per pulse at the sample plane. The sample was immobilized onto a quartz coverslip (Electron Microscope Sciences) via aminosilane functionalization ${ }^{106}$ and 2\% biotin-PEGylated surface. While imaging, the sample was immersed in an imaging buffer $(1 \times \mathrm{TAE}$ at final $\mathrm{pH}$ of 8.5$)$ containing triplet (Sigma-Aldrich; Trolox at $2 \mathrm{mM}$ final concentration) and enzymatic oxygen scavengers (protocatechuic acid/protocatechuic-3,4-dioxygenase at final concentrations of $2.5 \mathrm{mM} / 25 \mathrm{nM}$ ). The excitation laser was focused onto the sample and fluorescence was collected with the same oil-immersion objective (Olympus; UPLSAPO100XO, NA = 1.4). Single photons were collected by an avalanche photodiode (Excelitas; SPCM-AQRH-15) and arrival times were recorded by the same time-correlated single-photon counting module as used in ensemble fluorescence experiments. The instrument response function (IRF) was measured to be $\sim 380$ ps (full-width at half-maximum) by scattering excitation beam off a clean glass coverslip. All data analysis was performed in MATLAB (MathWorks, Inc.). Representative data and examples of data analysis are in Supplementary Information Section S4).

All-atom molecular dynamics simulations. The all-atom molecular dynamics simulations were performed using the program Amber16 ${ }^{107}$ with the OL15 force field ${ }^{108}$ for nucleic acids, and the GAFF2 force field $\frac{109}{}$ for the Cy3 molecules. Details of force field generation and all-atom system construction are given in Supplementary Information Sections S5.1 and S5.2. Periodic boundary conditions were applied in an orthogonal simulation cell, and the dynamics were integrated at a time step of $2 \mathrm{fs}$. Van der Waals energies were calculated using a 12 Å cutoff. The Particle Mesh Ewald (PME) method was used to calculate full electrostatics with a grid spacing of $1 \AA$ A . Full electrostatic forces and non-bonded forces were calculated at each time step ( $2 \mathrm{fs}$ ). These simulations were performed in the $N p T$ ensemble using a Berendsen barostat for pressure control at $1 \mathrm{bar}=\frac{110}{}$, and Langevin dynamics for temperature control at $300 \mathrm{~K}$ with a collision frequency of $5 \mathrm{ps}^{-1}[111$ Hydrogen atom bonds were constrained to their equilibrium lengths using the SHAKE algorithm $\frac{112}{12}$ during the simulations, and system configurations were recorded every 1 ps for downstream analysis. Prior to dynamics, energy minimization was performed on the solvent and ions alone, followed by the full unconstrained system for 10,000 steps each. Next, the solvent and ions were allowed to equilibrate as the system was heated to $300 \mathrm{~K}$ while the nucleic acid was harmonically constrained for $20 \mathrm{ps}$, followed by an unconstrained equilibration for 200 ps. For production dynamics, each system was run for $300 \mathrm{~ns}$ in duplicate (600 ns total). Structural analysis of MD simulations is outlined in Supplementary Information 
Section S5.5. Results highlighting distributions of Cy3 configurations, solvent accessible surface area, and orientation factors are shown in Supplementary Information Section S5.6.

Cy3 excited-state calculations. The position of the Cy3 molecules was sampled at every 2 ns, for 150 total configurations from each production MD simulation. The CPPTRAJ program 113 , as implemented in AmberTools 17 107 , was utilized to extract the Cy3 coordinate data from each trajectory. Next, the phosphate groups in the sampled $\mathrm{Cy} 3$ molecules were replaced with hydrogen atoms, and density functional theory (DFT) was used to first optimize the hydrogen atom positions followed by a time-dependent DFT calculation ${ }^{114} 115$ of the singlet excited states of the Cy3 molecules using the B3LYP functional ${ }^{116}$, the 6$31+\mathrm{G}(\mathrm{d})$ Gaussian basis set ${ }^{117}$, and implicit COSMO water solvation ${ }^{118}[119$, as implemented in the NWChem 6.6 software package ${ }^{120}$. Excited state energies from MD configurations are reported in Supplementary Figures S31 b, S32p, S33b, S34p, S35b, S36b, S37p, and S38b. Calculation of monomeric and dimeric Cy3 optical properties are shown in Supplementary Information Sections S5.7, S5.8, and S5.9.

Monte-Carlo FRET simulations All atom molecular dynamics simulation of the duplex and DX structure were performed using the AMBER Molecular Dynamics package and the parameters described in the Supplementary Information. Six replicates, each $100 \mathrm{~ns}$ in duration, were performed recording all atomic coordinates every 1 ps.

The data was analyzed using the CPPTraj software package ${ }^{\sqrt{113}}$ to extract the center of mass of each base pair and of the $\mathrm{Cy} 3$. The transition dipole moment unit vector of $\mathrm{Cy} 3$ was extracted as the unit vector from the N7 to the N17 atom. At each time point, and for each replicate the DNA axis was identified as the least squares fit of the base pair center of mass. The DNA axis was used to define the $\mathrm{z}$ axis in the right-handed DNA-centered coordinate system, while the $\mathrm{x}$ axis was defined in the direction between the DNA axis and Cy3 center of mass. Dipole orientation statistics in this local coordinate system were then sampled for both scaffolds. The anisotropy fluorescence decay experiments were then simulated by computing the orientation correlation function of the transition dipole moment $\frac{2}{5}\left(\frac{3}{2}\left\langle\cos \left(\theta_{t}\right)\right\rangle-\frac{1}{2}\right)$ where $\theta_{t}=\arccos \left(\hat{\mu}_{s} \cdot \hat{\mu}_{s+t}\right)$ is the angle between the transition dipole unit vectors $\hat{\mu}_{s}$ sampled a time $\mathrm{t}$ apart.

The position and orientation of the acceptor chromophore were obtained by assuming that the linker is 
always fully extended and the acceptor transition dipole is always parallel to the linker. These assumptions give rise to a cone of positions and orientations along a cone parallel to the axis of the DNA backbone, starting from the terminal base to which the acceptor is covalently bound. The direction of the linker axis was then sampled by selecting the polar angle from a normal distribution (mean $\bar{\theta}_{A}=0$, standard deviation $\sigma_{A}=$ 33) parametrized by the observed residual anisotropy, and by selecting the azimuthal angle from a uniform distribution. The polar angle was then rejected with a probability $P_{R}=\sin \theta$ to correctly weight the spherical samples. This algorithm yielded the same distribution of acceptor positions and orientations for both scaffoldings considered.

The donor chromophore position and orientation were sampled using two different models. In the first normal model, the position of the donor dye center of mass was taken to be fixed at its attachment position along the DNA scaffolding as a result of the double tether. Its orientation was then sampled, similarly to the acceptor, from a cone parallel to the DNA backbone axis. The polar angles were sampled from a normal distribution using the same algorithm as the acceptor with mean $\bar{\theta}_{D}=0$ and scaffolding dependent standard deviation $\left(\sigma_{\text {duplex }}=16^{\circ}, \sigma_{\mathrm{DX}}=22^{\circ}\right)$ and the azimuthal angle was sampled uniformly. The FRET efficiency and rate in this model was calculated for $10^{4}$ sampled donor and acceptor conformations. In the Molecular Dynamics model, the positions and orientations of the donor chromophore were instead sampled directly from the molecular dynamics simulation, sampling a donor and acceptor position for each frame.

To compute the FRET efficiency in the static limit, corresponding to an ensemble where chromophore reorientation occurs much slower than energy transfer, the FRET efficiency was computed for each conformation and then averaged to obtain the mean efficiency. The FRET efficiency in the dynamic limit, corresponding to a system where dipoles reorient much faster than energy transfer, is calculated by instead computing the FRET rate for each conformation. The average of this rate is then used to compute the overall efficiency of the FRET process.

Acknowledgements This work was partly funded by the National Science Foundation RAISE TAQS under Award Number 1839155 to M.B., A.P.W., and G.S.S.-C., the U.S. Department of Energy, Office of Basic Energy Sciences under Award Number DE-SC001999 to A.P.W., M.B., and G.S.S-C., the Army Research Office (ARO-MURI) Award Number W911NF1210420 to W.P.B. and M.B., the Office of Naval 
Research Award Number N00014-17-1-2609 to J.L.B. and M.B., the Office of Naval Research Award Numbers N00014-13-1-0664 and N00014- 15-1-2830 for funding the High-Performance Cluster used to perform simulations in this work. S.H. acknowledges an NSF Graduate Research Fellowship. W.J.C. acknowledges an NSERC Postgraduate Scholarship. G.S.S-C. acknowledges a Beckman Young Investigator Award, a Sloan Research Fellowship in Chemistry and a CIFAR Global Scholar Award. Financial support by the Swiss Nation Foundation (Grant Number: 200020_188468 to R.H.) is gratefully acknowledged.

Competing Interests The authors declare no competing financial interests.

Additional information * Correspondence and request for materials should be addressed to M.B. and G.S.S.-C. $†$ These authors contributed equally.

\section{References}

1. Blankenship, R. E. Molecular Mechanisms of Photosynthesis (John Wiley \& Sons, 2014).

2. van Grondelle, R., Bergström, H., Sundström, V. \& Gillbro, T. Energy transfer within the bacteriochlorophyll antenna of purple bacteria at $77 \mathrm{~K}$ studied by picosecond absorption recovery. Biochimica et Biophys. Acta 894, 313-326 (1987).

3. Cogdell, R. J., Gall, A. \& Köhler, J. The architecture and function of the light-harvesting apparatus of purple bacteria: from single molecules to in vivo membranes. Q. Rev. Biophys. 39, 227-324 (2006).

4. Scholes, G. D. \& Fleming, G. R. On the mechanism of light harvesting in photosynthetic purple bacteria: B800 to B850 energy transfer. J. Phys. Chem. B 104, 1854-1868 (2000).

5. Scholes, G. D. et al. Using coherence to enhance function in chemical and biophysical systems. Nature 543, 647-656 (2017).

6. Schaibley, J. R. et al. Valleytronics in 2D materials. Nat. Rev. Mater. 16055 (2016).

7. Noriega, R. et al. Manipulating excited-state dynamics of individual light-harvesting chromophores through restricted motions in a hydrated nanoscale protein cavity. J. Phys. Chem. B 119, 6963-6973 (2015). 
8. So, M. C., Wiederrecht, G. P., Mondloch, J. E., Hupp, J. T. \& Farha, O. K. Metal-organic framework materials for light-harvesting and energy transfer. Chem. Commun. 51, 3501-3510 (2015).

9. Scholes, G. D., Fleming, G. R., Olaya-Castro, A. \& van Grondelle, R. Lessons from nature about solar light harvesting. Nat. Chem. 3, 763-774 (2011).

10. Scholes, G. D. \& Rumbles, G. Excitons in nanoscale systems. Nat. Mater. 683-696 (2006).

11. Fleming, G. R., Schlau-Cohen, G. S., Amarnath, K. \& Zaks, J. Design principles of photosynthetic light-harvesting. Faraday Discuss. 155, 27-41 (2012).

12. Cheng, Y.-C. \& Fleming, G. R. Dynamics of light harvesting in photosynthesis. Annu. Rev. Phys. Chem. 60, 241-262 (2009).

13. Scholes, G. D., Fleming, G. R., Olaya-Castro, A. \& van Grondelle, R. Lessons from nature about solar light harvesting. Nat. Chem. 3, 763 (2011).

14. Krueger, B. P., Scholes, G. D. \& Fleming, G. R. Calculation of couplings and energy-transfer pathways between the pigments of LH2 by the ab initio transition density cube method. J. Phys. Chem. B 102, 5378-5386 (1998).

15. Jimenez, R., Dikshit, S. N., Bradforth, S. E. \& Fleming, G. R. Electronic excitation transfer in the LH2 complex of rhodobacter sphaeroides. J. Phys. Chem. 100, 6825-6834 (1996).

16. Wu, J., Liu, F., Shen, Y., Cao, J. \& Silbey, R. J. Efficient energy transfer in light-harvesting systems, I: optimal temperature, reorganization energy and spatial-temporal correlations. New J. Phys. 12, 105012 (2010).

17. Tiwari, V., Peters, W. K. \& Jonas, D. M. Electronic resonance with anticorrelated pigment vibrations drives photosynthetic energy transfer outside the adiabatic framework. Proc. Natl. Acad. Sci. 110, 1203-1208 (2013).

18. Ishizaki, A., Calhoun, T. R., Schlau-Cohen, G. S. \& Fleming, G. R. Quantum coherence and its interplay with protein environments in photosynthetic electronic energy transfer. Phys. Chem. Chem. Phys. 12, 7319-7337 (2010). 
19. Palmieri, B., Abramavicius, D. \& Mukamel, S. Lindblad equations for strongly coupled populations and coherences in photosynthetic complexes. J. Chem. Phys. 130, 204512 (2009).

20. Malỳ, P., Somsen, O. J., Novoderezhkin, V. I., Mančal, T. \& van Grondelle, R. The role of resonant vibrations in electronic energy transfer. ChemPhysChem 17, 1356-1368 (2016).

21. Saer, R. et al. Perturbation of bacteriochlorophyll molecules in Fenna-Matthews-Olson protein complexes through mutagenesis of cysteine residues. Biochimica et Biophys. Acta 1857, 1455-1463 (2016).

22. Maiuri, M., Ostroumov, E. E., Saer, R. G., Blankenship, R. E. \& Scholes, G. D. Coherent wavepackets in the Fenna-Matthews-Olson complex are robust to excitonic-structure perturbations caused by mutagenesis. Nat. Chem. 10, 177 (2018).

23. Fresch, E. et al. How the protein environment can tune the energy, the coupling, and the ultrafast dynamics of interacting chlorophylls: The example of the water-soluble chlorophyll protein. The $J$. Phys. Chem. Lett. 11, 1059-1067 (2020).

24. Yaghi, O. M. et al. Reticular synthesis and the design of new materials. Nature 423, 705-714 (2003).

25. Delor, M. et al. Exploiting chromophore-protein interactions through linker engineering to tune photoinduced dynamics in a biomimetic light-harvesting platform. J. Am. Chem. Soc. 140, 6278-6287 (2018).

26. Miller, R. A., Presley, A. D. \& Francis, M. B. Self-assembling light-harvesting systems from synthetically modified tobacco mosaic virus coat proteins. J. Am. Chem. Soc. 129, 3104-3109 (2007).

27. King, N. P. et al. Accurate design of co-assembling multi-component protein nanomaterials. Nature 510, 103-108 (2014).

28. Li, X., Sinks, L. E., Rybtchinski, B. \& Wasielewski, M. R. Ultrafast aggregate-to-aggregate energy transfer within self-assembled light-harvesting columns of zinc phthalocyanine tetrakis (perylenediimide). J. Am. Chem. Soc. 126, 10810-10811 (2004). 
29. Didraga, C., Klugkist, J. A. \& Knoester, J. Optical properties of helical cylindrical molecular aggregates: the homogeneous limit. J. Phys. Chem. B 106, 11474-11486 (2002).

30. Butkus, V., Valkunas, L. \& Abramavicius, D. Vibronic phenomena and exciton-vibrational interference in two-dimensional spectra of molecular aggregates. J. Chem. Phys. 140, 034306 (2014).

31. Lim, J. et al. Vibronic origin of long-lived coherence in an artificial molecular light harvester. Nat. Commun. 6, 7755 (2015).

32. Collini, E. \& Scholes, G. D. Coherent intrachain energy migration in a conjugated polymer at room temperature. Science 323, 369-373 (2009).

33. Hayes, D., Griffin, G. B. \& Engel, G. S. Engineering coherence among excited states in synthetic heterodimer systems. Science 340, 1431-1434 (2013).

34. Brown, K. E., Salamant, W. A., Shoer, L. E., Young, R. M. \& Wasielewski, M. R. Direct observation of ultrafast excimer formation in covalent perylenediimide dimers using near-infrared transient absorption spectroscopy. The J. Phys. Chem. Lett. 5, 2588-2593 (2014).

35. Halpin, A. et al. Two-dimensional spectroscopy of a molecular dimer unveils the effects of vibronic coupling on exciton coherences. Nat. Chem. 6, 196 (2014).

36. Wang, L. et al. Controlling quantum-beating signals in 2D electronic spectra by packing synthetic heterodimers on single-walled carbon nanotubes. Nat. Chem. 9, 219 (2017).

37. Cannon, B. L. et al. Large davydov splitting and strong fluorescence suppression: An investigation of exciton delocalization in dna-templated holliday junction dye aggregates. J. Phys. Chem. A 122, 2086-2095 (2018).

38. Cannon, B. L. et al. Coherent exciton delocalization in a two-state DNA-templated dye aggregate system. J. Phys. Chem. A 121, 6905-6916 (2017).

39. Kringle, L. et al. Temperature-dependent conformations of exciton-coupled Cy3 dimers in doublestranded DNA. J. Chem. Phys. 148, 085101 (2018). 
40. Cunningham, P. D. et al. Optical properties of vibronically coupled Cy3 dimers on DNA scaffolds. $J$. Phys. Chem. B 122, 5020-5029 (2018).

41. Mandal, S., Zhou, X., Lin, S., Yan, H. \& Woodbury, N. W. Directed energy transfer through DNAtemplated J-aggregates. Bioconjugate Chem. 30, 1870-1879 (2019).

42. Zhou, X. et al. Efficient long-range, directional energy transfer through DNA-templated dye aggregates. J. Am. Chem. Soc. 141, 8473-8481 (2019).

43. Boulais, É. et al. Programmed coherent coupling in a synthetic DNA-based excitonic circuit. Nat. Mater. 17, 159 (2018).

44. Banal, J. L., Kondo, T., Veneziano, R., Bathe, M. \& Schlau-Cohen, G. S. Photophysics of J-aggregatemediated energy transfer on DNA. J. Phys. Chem. Lett. 8, 5827-5833 (2017).

45. Mazuski, R. J. et al. Ultrafast excitation transfer in Cy5 DNA photonic wires displays dye conjugation and excitation energy dependency. J. Phys. Chem. Lett. 11, 4163-4172 (2020).

46. Adeyemi, O. O., Malinovskii, V. L., Biner, S. M., Calzaferri, G. \& Häner, R. Photon harvesting by excimer-forming multichromophores. Chem. Commun. 48, 9589-9591 (2012).

47. Probst, M., Wenger, D., Biner, S. M. \& Häner, R. The DNA three-way junction as a mould for tripartite chromophore assembly. Org. \& Biomol. Chem. 10, 755-759 (2012).

48. Hemmig, E. A. et al. Programming light-harvesting efficiency using DNA origami. Nano Lett. 16, 2369-2374 (2016).

49. Dutta, P. K. et al. DNA-directed artificial light-harvesting antenna. J. Am. Chem. Soc. 133, 1198511993 (2011).

50. Garo, F. \& Häner, R. A DNA-based light-harvesting antenna. Angewandte Chemie Int. Ed. 51, 916919 (2012).

51. Markova, L. I., Malinovskii, V. L., Patsenker, L. D. \& Häner, R. J-vs. H-type assembly: pentamethine cyanine (Cy5) as a near-IR chiroptical reporter. Chem. Commun. 49, 5298-5300 (2013). 
52. Cunningham, P. D., Díaz, S. A., Yurke, B., Medintz, I. L. \& Melinger, J. S. Delocalized two-exciton states in dna scaffolded cyanine dimers. The J. Phys. Chem. B (2020).

53. Heussman, D. et al. Measuring local conformations and conformational disorder of (cy3) 2 dimer labeled dna fork junctions using absorbance, circular dichroism and two-dimensional fluorescence spectroscopy. Faraday discussions 216, 211-235 (2019).

54. Cipolloni, M. et al. Coherent electronic and nuclear dynamics in a rhodamine heterodimer-dna supramolecular complex. Phys. Chem. Chem. Phys. 19, 23043-23051 (2017).

55. Sohail, S. H. et al. Dna scaffold supports long-lived vibronic coherence in an indodicarbocyanine (cy5) dimer. Chem. Sci. 11, 8546-8557 (2020).

56. Boulais, E. et al. Programmed coherent coupling in a synthetic dna-based excitonic circuit. Nat. Mater. 17, 159-166 (2018).

57. Nicoli, F. et al. Proximity-induced H-aggregation of cyanine dyes on dna-duplexes. J. Phys. Chem. A 120, 9941-9947 (2016).

58. Mustroph, H. et al. Relationship between the molecular structure of cyanine dyes and the vibrational fine structure of their electronic absorption spectra. ChemPhysChem 10, 835-840 (2009).

59. Hestand, N. J. \& Spano, F. C. Expanded theory of h-and j-molecular aggregates: the effects of vibronic coupling and intermolecular charge transfer. Chem. Rev. 118, 7069-7163 (2018).

60. Sundström, V. \& Gillbro, T. Excited state dynamics and photophysics of aggregated dye chromophores in solution. J. Chem. Phys. 83, 2733-2743 (1985).

61. Dean, J. C., Oblinsky, D. G., Rafiq, S. \& Scholes, G. D. Methylene blue exciton states steer nonradiative relaxation: Ultrafast spectroscopy of methylene blue dimer. J. Phys. Chem. B 120, 440-454 (2016).

62. Huff, J. et al. DNA-templated aggregates of strongly-coupled cyanine dyes: Nonradiative decay governs exciton lifetimes. J. Phys. Chem. Lett. (2019). 
63. Stennett, E. M., Ma, N., Van Der Vaart, A. \& Levitus, M. Photophysical and dynamical properties of doubly linked cy3-dna constructs. J. Phys. Chem. B 118, 152-163 (2013).

64. Arnott, S. et al. Dna secondary structures: helices, wrinkles, and junctions. In Cold Spring Harbor Symposia on Quantitative Biology, vol. 47, 53-65 (Cold Spring Harbor Laboratory Press, 1983).

65. Kjellberg, P., Brüggemann, B. \& Pullerits, T. Two-dimensional electronic spectroscopy of an excitonically coupled dimer. Phys. Rev. B 74, 024303 (2006).

66. Gallagher Faeder, S. M. \& Jonas, D. M. Two-dimensional electronic correlation and relaxation spectra: Theory and model calculations. J. Phys. Chem. A 103, 10489-10505 (1999).

67. Fuller, F. D. \& Ogilvie, J. P. Experimental implementations of two-dimensional Fourier transform electronic spectroscopy. Annu. Rev. Phys. Chem. 66, 667-690 (2015).

68. Szöcs, V. et al. Two-dimensional electronic spectra of symmetric dimers: Intermolecular coupling and conformational states. J. Chem. Phys. 124, 124511 (2006).

69. Cho, M., Vaswani, H. M., Brixner, T., Stenger, J. \& Fleming, G. R. Exciton analysis in 2d electronic spectroscopy. The J. Phys. Chem. B 109, 10542-10556 (2005).

70. Petkov, B. K. et al. Two-dimensional electronic spectroscopy reveals the spectral dynamics of förster resonance energy transfer. Chem 5, 2111-2125 (2019).

71. Rothemund, P. W. Folding dna to create nanoscale shapes and patterns. Nature 440, 297-302 (2006).

72. Douglas, S. M. et al. Self-assembly of dna into nanoscale three-dimensional shapes. Nature 459, 414-418 (2009).

73. Veneziano, R. et al. Designer nanoscale dna assemblies programmed from the top down. Science 352, 1534-1534 (2016).

74. Jun, H. et al. Autonomously designed free-form 2D DNA origami. Sci. Adv. 5, eaav0655 (2019).

75. Jun, H. et al. Automated sequence design of 3D polyhedral wireframe DNA origami with honeycomb edges. ACS Nano 13, 2083-2093 (2019). 
76. Jun, H., Wang, X., Bricker, W. P. \& Bathe, M. Automated sequence design of $2 \mathrm{~d}$ wireframe dna origami with honeycomb edges. Nat. Commun. 10, 1-9 (2019).

77. Wamhoff, E.-C. et al. Programming structured dna assemblies to probe biophysical processes. Annu. Rev. Biophys. 48, 395-419 (2019).

78. Li, X., Yang, X., Qi, J. \& Seeman, N. C. Antiparallel dna double crossover molecules as components for nanoconstruction. J. Am. Chem. Soc. 118, 6131-6140 (1996).

79. Sa-Ardyen, P., Vologodskii, A. V. \& Seeman, N. C. The flexibility of DNA double crossover molecules. Biophys. J. 84, 3829-3837 (2003).

80. Kwak, K., Park, S., Finkelstein, I. J. \& Fayer, M. Frequency-frequency correlation functions and apodization in two-dimensional infrared vibrational echo spectroscopy: A new approach. J. Chem. Phys. 127, 124503 (2007).

81. Sanda, F., Perlík, V., Lincoln, C. N. \& Hauer, J. Center line slope analysis in two-dimensional electronic spectroscopy. J. Phys. Chem. A 119, 10893-10909 (2015).

82. Nemeth, A. et al. Vibrational wave packet induced oscillations in two-dimensional electronic spectra. I. experiments. J. Chem. Phys. 132, 184514 (2010).

83. Mančal, T. et al. Vibrational wave packet induced oscillations in two-dimensional electronic spectra. II. theory. J. Chem. Phys. 132, 184515 (2010).

84. Nowakowski, P. J., Khyasudeen, M. F. \& Tan, H.-S. The effect of laser pulse bandwidth on the measurement of the frequency fluctuation correlation functions in 2D electronic spectroscopy. Chem. Phys. 515, 214-220 (2018).

85. Khyasudeen, M. F., Nowakowski, P. J. \& Tan, H.-S. Measuring the ultrafast correlation dynamics between the Qx and Qy bands in chlorophyll molecules. J. Phys. Chem. B 123, 1359-1364 (2019).

86. Hart, S. M., Banal, J. L., Bathe, M. \& Schlau-Cohen, G. Identification of non-radiative decay pathways in Cy3. J. Phys. Chem. Lett. (accepted). 
87. Alden, C. J. \& Kim, S.-H. Solvent-accessible surfaces of nucleic acids. J. Mol. Biol. 132, 411-434 (1979).

88. Sanborn, M. E., Connolly, B. K., Gurunathan, K. \& Levitus, M. Fluorescence properties and photophysics of the sulfoindocyanine Cy3 linked covalently to dna. J. Phys. Chem. B 111, 11064-11074 (2007).

89. Weinreis, S. A., Ellis, J. P. \& Cavagnero, S. Dynamic fluorescence depolarization: a powerful tool to explore protein folding on the ribosome. Methods 52, 57-73 (2010).

90. Förster, T. Zwischenmolekulare energiewanderung und fluoreszenz. Annalen der physik 437, 55-75 (1948).

91. Van Der Meer, B. W., Coker, G. \& Chen, S.-Y. S. Resonance Energy Transfer: Theory and Data (Wiley-VCH, 1994).

92. Dale, R. E., Eisinger, J. \& Blumberg, W. The orientational freedom of molecular probes. the orientation factor in intramolecular energy transfer. Biophys. J. 26, 161-193 (1979).

93. Stryer, L. Fluorescence energy transfer as a spectroscopic ruler. Annu. review biochemistry 47, 819846 (1978).

94. VanBeek, D. B., Zwier, M. C., Shorb, J. M. \& Krueger, B. P. Fretting about FRET: Correlation between $\kappa$ and r. Biophys. J. 92, 4168-4178 (2007).

95. Eilert, T., Kallis, E., Nagy, J., Röcker, C. \& Michaelis, J. Complete kinetic theory of FRET. J. Phys. Chem. B 122, 11677-11694 (2018).

96. Sindbert, S. et al. Accurate distance determination of nucleic acids via Förster resonance energy transfer: implications of dye linker length and rigidity. J. Am. Chem. Soc. 133, 2463-2480 (2011).

97. Iqbal, A. et al. Orientation dependence in fluorescent energy transfer between cy 3 and cy 5 terminally attached to double-stranded nucleic acids. Proc. Natl. Acad. Sci. 105, 11176-11181 (2008).

98. Ivanov, V., Li, M. \& Mizuuchi, K. Impact of emission anisotropy on fluorescence spectroscopy and fret distance measurements. Biophys. J. 97, 922-929 (2009). 
99. Chen, X. \& Silbey, R. J. Effect of correlation of local fluctuations on exciton coherence. J. Chem. Phys. 132, 204503 (2010).

100. Chen, X. \& Silbey, R. J. Excitation energy transfer in a non-markovian dynamical disordered environment: Localization, narrowing, and transfer efficiency. The J. Phys. Chem. B 115, 5499-5509 (2011).

101. Strümpfer, J. \& Schulten, K. The effect of correlated bath fluctuations on exciton transfer. The J. Chem. Phys. 134, 03B603 (2011).

102. Delor, M. et al. Exploiting chromophore-protein interactions through linker engineering to tune photoinduced dynamics in a biomimetic light-harvesting platform. J. Am. Chem. Soc. doi: 10.1021/jacs.7b13598 (2018).

103. Würth, C., Grabolle, M., Pauli, J., Spieles, M. \& Resch-Genger, U. Relative and absolute determination of fluorescence quantum yields of transparent samples. Nat. Protoc. 8, 1535 (2013).

104. Son, M., Mosquera-Vázquez, S. \& Schlau-Cohen, G. S. Ultrabroadband 2D electronic spectroscopy with high-speed, shot-to-shot detection. Opt. Express 25, 18950-18962 (2017).

105. Son, M., Pinnola, A., Bassi, R. \& Schlau-Cohen, G. S. The electronic structure of Lutein 2 is optimized for light harvesting in plants. Chem 5, 575-584 (2019).

106. Hua, B. et al. An improved surface passivation method for single-molecule studies. Nat. Methods 11, 1233 (2014).

107. Case, D. et al. AMBER 2017 (University of California, San Francisco, 2017).

108. Galindo-Murillo, R. et al. Assessing the current state of Amber force field modifications for DNA. $J$. Chem. Theory Comput. 12, 4114-4127 (2016).

109. Wang, J., Wolf, R. M., Caldwell, J. W., Kollman, P. A. \& Case, D. A. Development and testing of a general Amber force field. J. Comput. Chem. 25, 1157-1174 (2004).

110. Berendsen, H., Postma, J., van Gunsteren, W., DiNola, A. \& Haak, J. Molecular dynamics with coupling to an external bath. J. Chem. Phys. 81, 3684-3890 (1984). 
111. Izaguirre, J. A., Catarello, D. P., Wozniak, J. M. \& Skeel, R. D. Langevin stabilization of molecular dynamics. J. Chem. Phys. 114, 2090-2098 (2000).

112. Ryckaert, J.-P., Ciccotti, G. \& Berendsen, H. J. C. Numerical integration of the Cartesian equations of motion of a system with constraints: Molecular dynamics of n-alkanes. J. Comput. Phys. 23, 327-341 (1977).

113. Roe, D. R. \& Cheatham, T. E., III. PTRAJ and CPPTRAJ: Software for processing and analysis of molecular dynamics trajectory data. J. Chem. Theory Comput. 9, 3084-3095 (2013).

114. Kohn, W. \& Sham, L. J. Self-consistent equations including exchange and correlation effects. Phys. Rev. 140, A1133-A1138 (1965).

115. Runge, E. \& Gross, E. K. U. Density-functional theory for time-dependent systems. Phys. Rev. Lett. 52, 997-1000 (1984).

116. Becke, A. D. Density-functional thermochemistry. III. the role of exact exchange. J. Chem. Phys. 98, $5648-5652$ (1992).

117. Hehre, W. J., Ditchfield, R. \& Pople, J. A. Self-consistent molecular orbital methods. XII. further extensions of gaussian-type basis sets for use in molecular orbital studies of organic molecules. $J$. Chem. Phys. 56, 2257-2261 (1972).

118. Klamt, A. \& Schüürmann, G. COSMO: A new approach to dielectric screening in solvents with explicit expressions for the screening energy and its gradient. J. Chem. Soc. Perkin Transactions 2 799-805 (1993).

119. York, D. M. \& Karplus, M. A smooth solvation potential based on the conductor-like screening model. J. Phys. Chem. A 103, 11060-11079 (1999).

120. Valiev, M. et al. NWChem: A comprehensive and scalable open-source solution for large scale molecular simulations. Comput. Phys. Commun. 181, 1477-1489 (2010). 